\title{
High-order harmonic generation in the presence of a resonance
}

\author{
Maria Tudorovskaya ${ }^{1,2, *}$ and Manfred Lein ${ }^{1}$ \\ ${ }^{1}$ Institut für Theoretische Physik and Centre for Quantum Engineering and Space-Time Research (QUEST), Leibniz Universität Hannover, \\ Appelstraße 2, D-30167 Hannover, Germany \\ ${ }^{2}$ Institut für Physik, Universität Kassel, Heinrich-Plett-Straße 40, D-31432 Kassel, Germany
}

(Received 9 May 2011; published 29 July 2011)

\begin{abstract}
We investigate high-order harmonic generation from laser-irradiated systems that support a shape resonance. From the numerical solution of the time-dependent Schrödinger equation, we calculate the harmonic spectra and the time-frequency analysis of the harmonic intensity and phase. The analysis reveals the separate contributions of the short and long trajectories as well as the resonance. A range of harmonics is strongly enhanced by the presence of the resonance irrespective of the pulse length. The signature of the resonance remains significant after coherent summation over intensities as a simple method to simulate macroscopic effects. The time-frequency analysis supports the recently proposed four-step mechanism of the enhanced harmonic generation process.
\end{abstract}

DOI: 10.1103/PhysRevA.84.013430

PACS number(s): $33.80 . R v, 42.65 . K y$

\section{INTRODUCTION}

High-order harmonic generation (HHG) is a nonlinear process occuring in intense laser fields acting on various types of targets [1-4]. It causes the emission of extreme ultraviolet (XUV) coherent radiation. In these experiments, the typical order of the laser intensity is $10^{14} \mathrm{~W} / \mathrm{cm}^{2}$. HHG can be understood in a simple three-step model [5]. The first step is ionization of the target by the laser field. Second, the electron is driven by the oscillating laser field and returns to the core. The last step is radiative recombination resulting in the emission of a high-harmonic photon. The emitted HHG spectrum exhibits a plateau with a cutoff at the energy $3.17 U_{p}+I_{p}$ [5], where $U_{p}$ is the ponderomotive potential and $I_{p}$ is the ionization potential of the target. For harmonic frequencies below the cutoff, there exist short and long trajectories [6], i.e. there are two different durations of electron trajectories contributing to the same harmonic frequency with return times in the same half cycle. These trajectories can be approximately obtained from the classical equation of motion for the second step of the three-step model. The presence of the nearly classical trajectories has been confirmed by the measurement of the attosecond chirp [7]. The three-step sequence is repeated in every half cycle of the laser field. This periodicity leads to the formation of odd-order harmonic radiation unless the laser pulse is extremely short.

The recombination step in HHG is the inverse process of one-photon photoionization. The dependence of the recombination probability on the electron return energy and on the structure of the target is reflected in the HHG spectrum and has been the subject of intensive research in the past years. For example, the orientation dependence of HHG from aligned molecules [8-11] has led to imaging of molecular orbitals $[12,13]$. In molecules, significant contributions to the HHG signal may arise not only from the outermost bound electron orbital but also from lower-lying orbitals [14-16]. However, the experimental findings were successfully reproduced also by the quantitative rescattering theory $[17,18]$, which does

\footnotetext{
"maria.tudorovskaya@itp.uni-hannover.de
}

not resort to lower-lying orbitals but treats the recombination step very accurately by using the exact recombination cross sections. For atoms, the accepted view is that only the outermost orbital is relevant for HHG and therefore a direct link between HHG and valence-shell photoionization can be made. For example, the Cooper minimum in the photoionization cross section of argon atoms [19] is found also in HHG spectra from argon [20]. Hence, to enhance the notoriously low efficiency of the HHG process, it appears promising to exploit the effect of resonances, which are known to be of great importance in photoionization. The investigation of resonant peaks in the photoionization cross section has a long history, including studies of autoionization resonances [21,22], shape resonances [23], and giant resonances [24], but there have been only a few studies on the role of resonances in HHG. Note that the previous discussions of multiphoton resonances in HHG [25-27] refer to a different physical situation, namely, the resonant excitation of bound states, which has been made responsible also for the strong enhancement of groups of peaks in above-threshold ionization spectra [28]. In contrast, here we discuss resonances in the meaning of metastable states embedded in the electronic continuum of the target system.

Experimentally, resonant enhancement of single harmonics has been observed using many-cycle laser pulses interacting with plasma plumes, see the review [4] and references therein. It has been argued that these findings are due to the population of metastable states via a resonant excitation mechanism [29-31]. The phase-matching aspects of this emission mechanism were discussed in [31,32]. Recently, a four-step model was suggested by Strelkov [33] to explain the resonant HHG. According to the model, the last step of the usual HHG process (radiative transition to the ground state) is split into two steps: the returning electron is trapped by the target into the resonance and then it relaxes to the ground state emitting the XUV photon. Although the results of Strelkov's theoretical analysis show agreement with the experiment, it remains to be clarified whether the four-step process really occurs in the time evolution obtained from the time-dependent Schrödinger equation (TDSE).

In the present article we analyze the effect of a shape resonance on HHG. A shape resonance is a one-electron 
phenomenon occurring when the potential exhibits a barrier supporting a metastable state. In fact, a potential with a shape resonance was used in [33] to mimic the autoionizing states of the ions relevant for the experimentally observed HHG in plasmas [4]. Shape resonances may also be important for understanding molecular HHG spectra, e.g., from $\mathrm{N}_{2}$ [17]. We investigate both the single-atom response and phase-matching aspects [34]. The four-step mechanism is revealed by the time-frequency analysis of the harmonic radiation. Insight is gained also by applying the time-frequency analysis to the harmonic phase.

\section{METHOD}

Atomic units are used throughout the paper. We numerically solve the one-dimensional (1D) time-dependent Schrödinger equation in the presence of a laser field

$$
i \frac{\partial \Psi(x, t)}{\partial t}=-\frac{1}{2} \frac{\partial^{2} \Psi(x, t)}{\partial x^{2}}+\left[V_{0}(x)+E(t) x\right] \Psi(x, t),
$$

where $V_{0}(x)$ is the potential of the unperturbed system and $E(t)$ is the laser field. We use a model potential $V_{0}(x)$ with a barrier

$$
\begin{aligned}
V_{0}(x)= & -\alpha+\frac{\alpha}{1+e^{\frac{x+\beta}{\gamma}}}+\frac{\alpha}{1+e^{\frac{-x+\beta}{\gamma}}}+\frac{\delta}{\left(\epsilon+x^{2}\right)\left(1+e^{\frac{x+\beta}{\gamma}}\right)} \\
& +\frac{\delta}{\left(\epsilon+x^{2}\right)\left(1+e^{\frac{-x+\beta}{\gamma}}\right)} .
\end{aligned}
$$

Figure 1(a) shows the two potential shapes that we use throughout this article, denoted in the following as potential 1 and potential 2. The parameters are $\alpha_{1}=1.32, \beta_{1}=1.27, \gamma_{1}=$ $0.23, \delta_{1}=9.5, \epsilon_{1}=1.4$ for potential 1 and $\alpha_{2}=1.5, \beta_{2}=$ 1.27, $\gamma_{2}=0.23, \delta_{2}=14, \epsilon_{2}=1.4$ for potential 2. The TDSE is solved by means of the split-operator technique $[35,36]$ and the ground-state wave function $\Psi_{0}(x)$ is obtained by imaginary-time propagation [37].

The potential barrier leads to the existence of a shape resonance, i.e., a metastable state corresponding to an electron trapped in the interior region with a finite lifetime. The
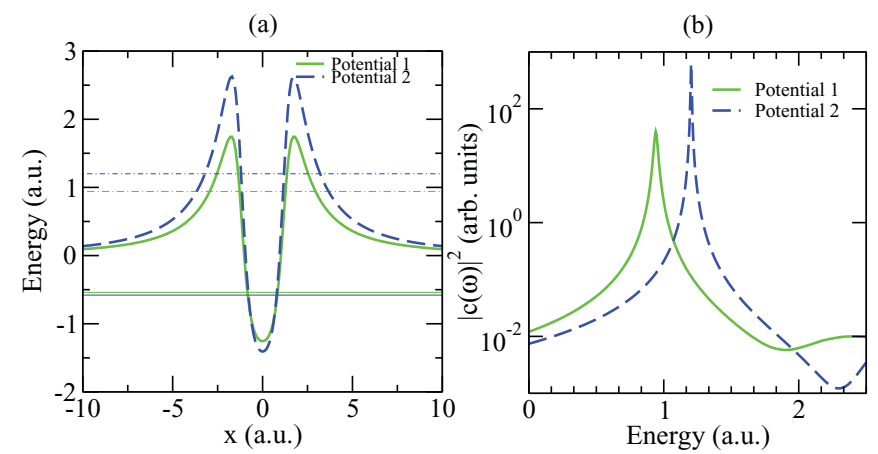

FIG. 1. (Color online) (a) The two potentials used in this work. The ground-state energies of potentials 1 and 2 are at -0.54 and -0.58 a.u., as indicated (solid horizontal lines). Shape resonances are found to be at 0.95 and 1.20 a.u., respectively (dot-dashed horizontal lines). (b) Modulus squared of the Fourier-transformed autocorrelation functions showing the resonances of potentials 1 and 2.

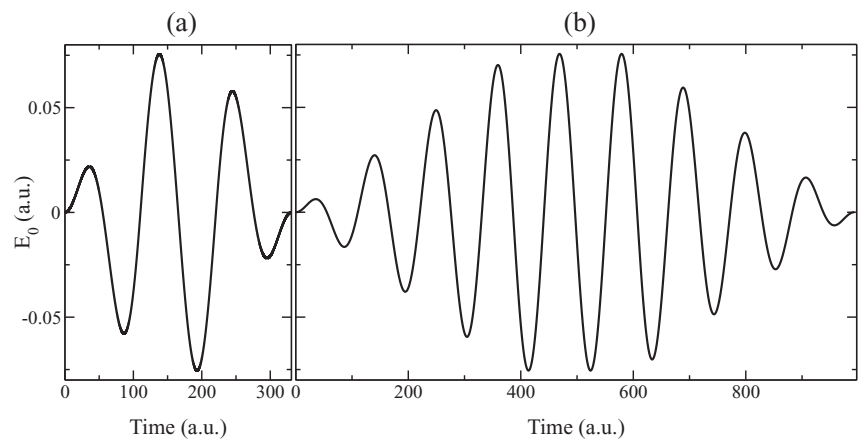

FIG. 2. (a) Short pulse with a (1-1-1) envelope. (b) Long pulse with a (3.5-2-3.5) envelope.

resonance energy can be obtained by inspection of the modulus squared of the Fourier-transformed autocorrelation function $c(t)=\left\langle\Phi_{0} \mid \Phi(t)\right\rangle$, where $\Phi(x, t)$ is the result of propagation of an arbitrary function $\Phi_{0}(x)$ that has overlap with the resonant state. We choose $\Phi_{0}(x)=\Phi_{G}(x)-\left\langle\Psi_{0} \mid \Phi_{G}\right\rangle \Psi_{0}(x)$ with a Gaussian wave packet $\Phi_{G}(x)=\exp \left[-(x-0.9)^{2}\right]$, so that the ground state does not disturb the resulting picture. The result clearly shows the positions of the resonance for the two potentials, see Fig. 1(b). The lifetimes are obtained from the widths of the resonance peaks. By fitting the peaks to the Breit-Wigner formula [38], we obtain the values $\Gamma_{1}=0.030$ and $\Gamma_{2}=0.0071$ a.u. This means that the decay time of the resonance in potential 1 is shorter than the optical cycle of an $800-\mathrm{nm}$ laser field, while for potential 2 the resonance is longer lived than the optical cycle.

A laser field of the form $E(t)=E_{0} \sin \left(\omega_{L} t\right) f(t)$ is used, where $E_{0}$ and $\omega_{L}$ are the field amplitude and frequency and $f(t)$ is a trapezoidal envelope. We employ a "short" and a "long" laser pulse as shown in Fig. 2. The short pulse has a (1-1-1) shape, i.e., one optical cycle switch-on and switch-off times and a one-cycle plateau. The long pulse has a (3.5-2-3.5) shape. All calculations are done for an $800-\mathrm{nm}$ wavelength.

The dipole acceleration is calculated according to

$$
a(t)=\left\langle\Psi(t)\left|\frac{d V_{0}(x)}{d x}+E(t)\right| \Psi(t)\right\rangle,
$$

and the HHG spectrum is proportional to

$$
S(\omega)=\left|\int d t a(t) e^{i \omega t}\right|^{2} .
$$

We investigate the time-frequency distribution of the harmonics using the Gabor transform $[39,40]$

$$
G(\omega, t)=\int d \tau a(\tau) e^{i \omega \tau} e^{-(t-\tau)^{2} / 2 \sigma^{2}}
$$

We use the parameter $\sigma=1 /\left(3 \omega_{L}\right)$. The modulus squared of $G(\omega, t)$ is the time-frequency distribution of the harmonic intensity. We analyze also the phase of $G(\omega, t)$, which gives insight into the time-frequency aspects of phase matching.

In a real HHG experiment, the macroscopic propagation of harmonics emitted by different atoms of a medium plays 
(a)

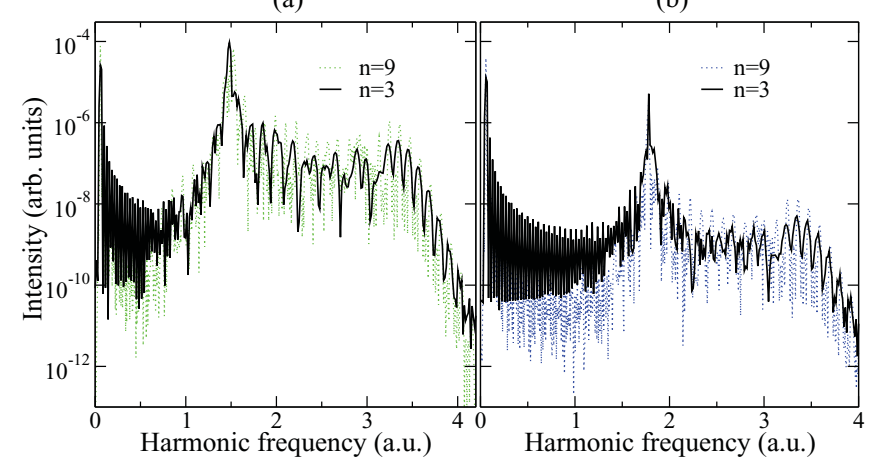

FIG. 3. (Color online) HHG spectra for (a) potential 1 and (b) potential 2, calculated with different pulse lengths for the potentials shown in Fig. 1(a). $n$ is the number of optical cycles in a pulse with intensity $I=4 \times 10^{14} \mathrm{~W} / \mathrm{cm}^{2}$.

an important role [34]. Being distributed in space, different atoms are exposed to different intensities of the laser field. We simulate the phase matching by coherent summation over intensities in an appropriate range $\Delta I[41,42]$ in order to study the effect on the HHG spectrum and time-frequency distribution. We calculate the total effective dipole acceleration generated by $N$ uniformly distributed intensities in the range $\Delta I$ according to

$$
a_{\mathrm{eff}}(t)=\sum_{i=1}^{N} a_{I_{i}} / N .
$$

The results from the intensity-averaging procedure agree with the results from the more complicated macroscopic propagation for the frequently used experimental configuration with the gas jet after the focus [42]. This configuration favors the selection of the short trajectories [43].

\section{SINGLE-ATOM RESPONSE}

In this section, we address the question of whether the HHG spectrum calculated from the TDSE for a single atom shows the signature of the shape resonances. The mechanism of resonant HHG is investigated by inspection of the timefrequency analysis.

\section{A. HHG spectra}

We show in Fig. 3(a) the HHG spectra calculated for different pulse lengths and for the two different potentials. The cutoffs are in agreement with the classical formula and, as expected, the harmonic peaks become sharper with increasing pulse length. In all cases, the spectrum exhibits a strong peak at the harmonic frequency that corresponds to the difference between the resonance energy and the ground-state energy. At harmonic orders below the resonance peak, harmonic emission appears to be strongly suppressed. This is an immediate consequence of the potential barrier making it almost impossible for slow electrons to return to the bound-state region. The enhancement due to the resonance is two to three orders of magnitude. Moreover, the calculation shows that the resonance is observed irrespective of the pulse length. This indicates that the population of the resonance builds up very quickly regardless of the lifetime or pulse duration. This supports the interpretation in terms of the four-step model rather than a resonant excitation mechanism. In all the following calculations, we employ the long pulse shown in Fig. 2(b)

\section{B. Time-frequency analysis}

The time-frequency distribution is calculated from the Gabor transform, Eq. (5). The full picture shows a periodic repetition of arches comprising the long and short trajectories, due to the periodic character of the laser field. We plot sections containing about three half cycles in Figs. 4(a) and 4(b). One can clearly see that the main contribution to the high-frequency part of the spectra is due to the long trajectories. This is a known effect in 1D TDSE calculations; in 3D TDSE calculations, the radiation is dominated by the short trajectories [40]. The figures show that the resonance causes a strong signal around a fixed frequency. This type of emission dominates the spectra at harmonic frequencies below 2 a.u. It is striking that the emission at the resonance increases whenever a long-trajectory branch crosses the resonance emission frequency. It means that (a)

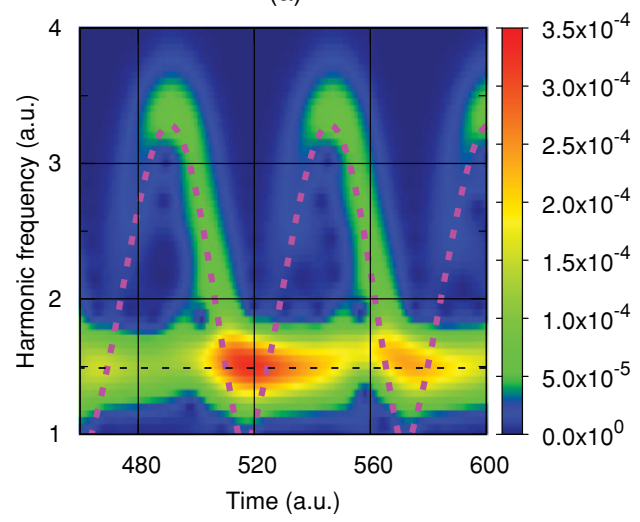

(b)

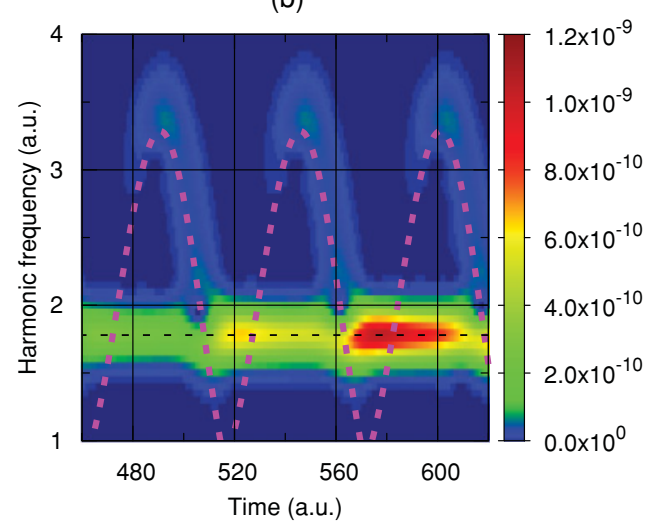

FIG. 4. (Color online) Time-frequency distribution of the harmonic radiation for (a) potential 1 and (b) potential 2 . The laser intensity is $4 \times 10^{14} \mathrm{~W} / \mathrm{cm}^{2}$. The thick dashed purple curves show classical trajectories for a monochromatic field at $I=4 \times 10^{14} \mathrm{~W} / \mathrm{cm}^{2}$. The black dashed lines show the positions of the resonances. 
(a)

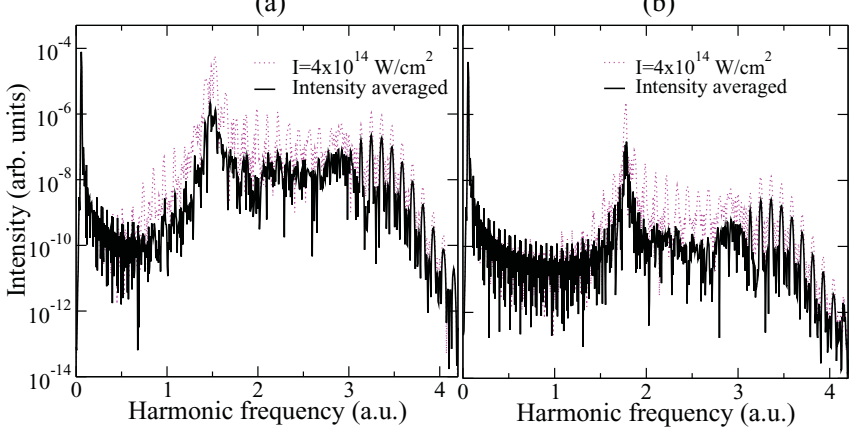

FIG. 5. (Color online) HHG spectra for a single atom (dashed curve) and after coherent summation over intensities (solid curve). (a) Potential 1. (b) Potential 2.

the resonance radiates more intensely after the electron returns to the core. We conclude that the returning electron is captured to the resonance state in agreement with Strelkov's four-step model [33]. The comparison between different half cycles shows that potential 1 behaves differently from potential 2 . We suggest the following explanation. The lifetime of potential 1 is $1 / \Gamma_{1}=34$ a.u., so that the resonance has decayed by the time of the next electron return. In potential 2 the resonance survives for a longer time since $1 / \Gamma_{2}=141$ a.u. It does not decay during a half cycle of the laser pulse, so the population of the resonance can potentially be increased every half cycle.

An increase of the resonance population is not clearly seen when the short-trajectory branch crosses the resonance emission frequency. We believe that this contribution exists, but it is simply hidden under the strong resonance signal from the long trajectory.

\section{PHASE MATCHING}

\section{A. Intensity-averaged HHG spectra}

The macroscopic effects on the HHG spectra are simulated by coherent summation over an intensity interval. We use 31 intensities in the range from $3.85 \times 10^{14}$ to $4.15 \times 10^{14} \mathrm{~W} / \mathrm{cm}^{2}$. Figure 5 shows the comparison of the single-atom response spectra for the laser intensity $4 \times 10^{14} \mathrm{~W} / \mathrm{cm}^{2}$ and the intensity-averaged HHG spectra. The intensity averaging leads to a substantial suppression of the spectrum in the plateau region due to the phase mismatch between different intensities. Obviously, the resonance remains significant for both considered potentials.

\section{B. Intensity averaging and time-frequency analysis}

In order to explain the intensity-averaged HHG spectra, we investigate the harmonic phases in detail. At fixed laser intensity we calculate the phase of the Gabor transform $G(\omega, t)$ from Eq. (5). We then calculate the phase difference $\delta \phi$ of the same harmonic generated by two different laser pulses with slightly different intensities $I_{1}$ and $I_{2}$. Small $\delta \phi$ implies good phase matching while large $\delta \phi$ leads to phase mismatch.

The result for $I_{1}=4.14 \times 10^{14}$ and $I_{2}=4.15 \times$ $10^{14} \mathrm{~W} / \mathrm{cm}^{2}$ is shown in Fig. 6. The shown time interval covers about one half cycle of the laser field. One can see that the phase matching for the short trajectory (roughly the left half of the diagrams) in the plateau is better than the phase matching for the long trajectory (right half of the diagrams). We have confirmed that this holds also for other intensities in the range used for the intensity averaging. We thus expect that intensity averaging favors the short trajectories and suppresses the long trajectories. In the region of the resonance, we find a large phase difference comparable to the long-trajectory phase difference. However, it is likely that a contribution from the resonance with better phase matching is not visible in these plots. This is because the calculated emission is dominated by the population of the resonance due to the long-trajectory returns, see the time-frequency analysis in Sec. III B. If the resonance is populated by trapping a returning electron, we expect that the phase of the emission is locked to the phase of the returning electron. In fact, we clearly see in Fig. 6 that the phase difference varies smoothly as the long-trajectory branch merges into the resonance around the time 510 a.u. Thus, if an electron returns on the short trajectory and gets trapped in the resonance, the emission should be well phase matched, as is the case for the normal short-trajectory radiation. (a)

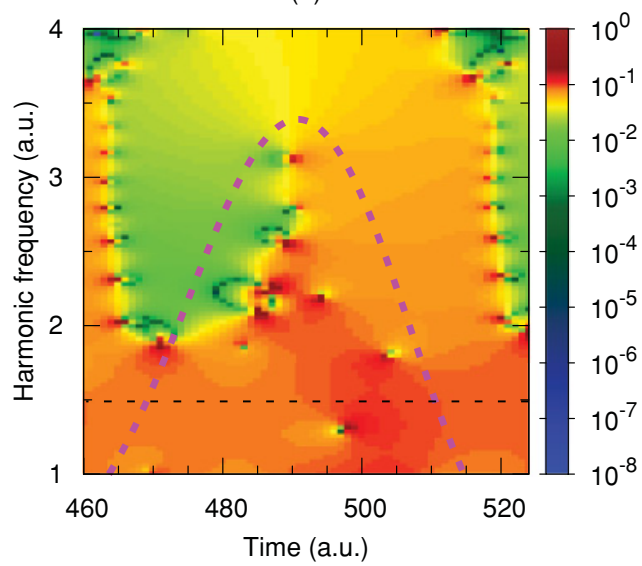

(b)

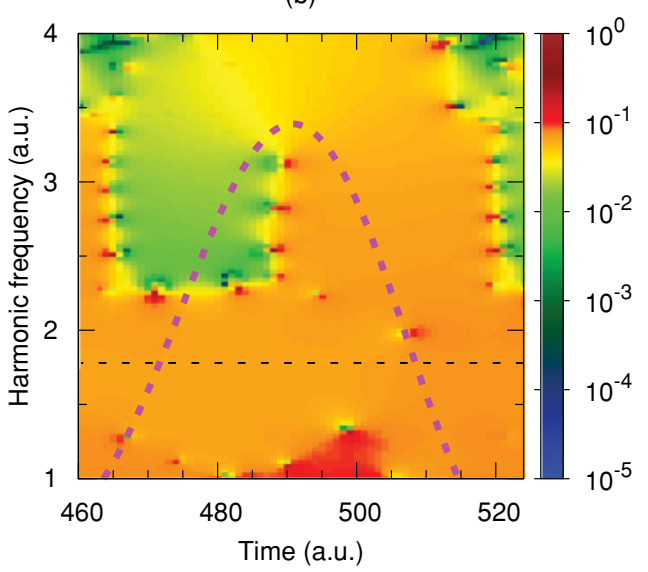

FIG. 6. (Color online) Time-frequency analysis of the harmonic phase difference between the intensities $I_{1}=4.14 \times 10^{14}$ and $I_{2}=$ $4.15 \times 10^{14} \mathrm{~W} / \mathrm{cm}^{2}$. (a) Potential 1. (b) Potential 2. The thick dashed purple curves show classical trajectories at $I=4.15 \times 10^{14} \mathrm{~W} / \mathrm{cm}^{2}$. The black dashed lines show the positions of the resonances. 
(a)

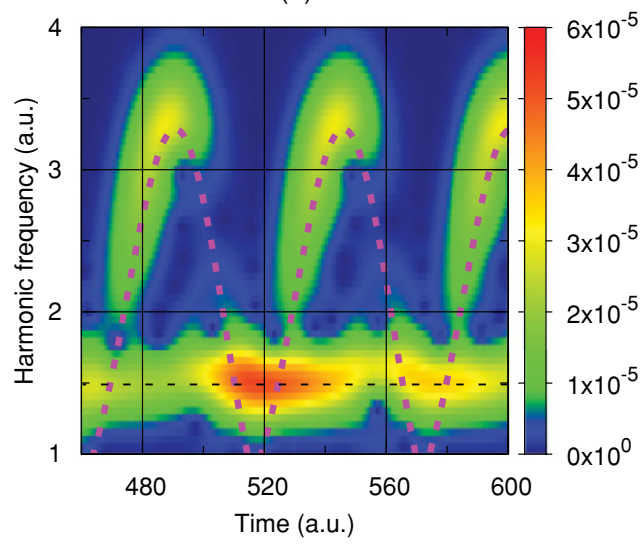

(b)

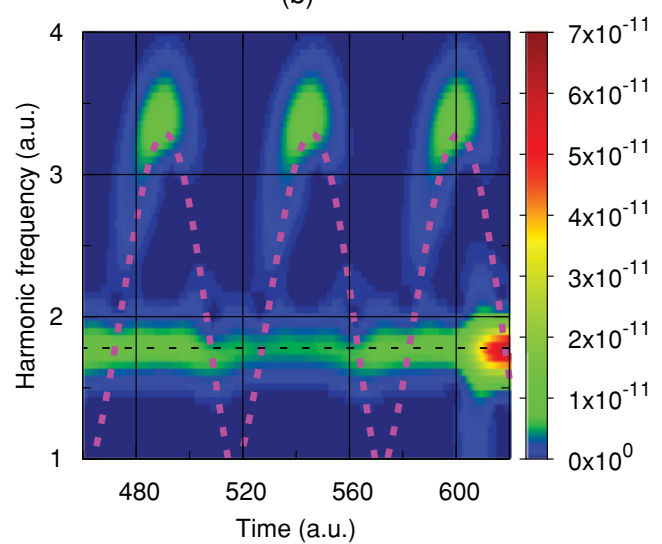

FIG. 7. (Color online) Time-frequency distribution of the harmonic radiation after intensity averaging. (a) Potential 1. (b) Potential 2. The thick dashed purple curves show classical trajectories for a monochromatic field at $I=4 \times 10^{14} \mathrm{~W} / \mathrm{cm}^{2}$. The black dashed lines show the positions of the resonances.

Figure 7 shows the Gabor analysis for the harmonic intensity after summation over intensities. As expected, the intensity averaging effectively selects the contributions with good phase matching: the emission from the short-trajectory branches is much more intense than from the long-trajectory branches. The signature of the resonance remains significant for both potentials. The detailed behavior depends on the lifetime. For a long-lived resonance (potential 2), the addition of the newly trapped population to the already existing population can be constructive or destructive depending on the relative phase. Figure 7(b) shows an example where the electron return leads to a population reduction, see the decrease of harmonic emission at the time 510 a.u. Apparently, XUV radiation with very interesting steplike temporal profiles can be generated in this way. The short-lived resonance (potential 1) decays substantially before the next return of an electron, and therefore Fig. 7(a) does not show such a cancellation effect.

\section{CONCLUSION}

We have analyzed HHG in the presence of a shape resonance. To understand the HHG mechanism, we investigated the time-frequency analysis of the intensity and phase. We have found that the resonance gives rise to a clear signature in the HHG spectrum irrespective of the pulse length. The time-frequency analysis supports Strelkov's four-step model, according to which the recombination process consists of two steps: capture of the returning electron into the resonance and subsequent radiative transition to the ground state. While the present 1D calculation favors capture from the long trajectory, we expect that a full 3D calculation will show a similar mechanism, but with higher weight given to the short trajectory. By the nature of this process, the emitted harmonic radiation is phase-locked with the usual harmonic emission from the short and long trajectories. For longlived resonances, interference occurs between the populations caused by recollisions in different half cycles. This leads to new possibilities for XUV pulse shaping on the subfemtosecond time scale.

\section{ACKNOWLEDGMENTS}

We acknowledge the support from the European Marie Curie Initial Training Network Grant No. CA-ITN-214962FASTQUAST. We thank the Deutsche Forschungsgemeinschaft for funding the Centre for Quantum Engineering and Space-Time Research (QUEST).
[1] A. McPherson et al., J. Opt. Soc. Am. B 4, 595 (1987).

[2] M. Ferray, A. L'Huillier, X. F. Li, L. A. Lompre, G. Mainfray, and C. Manus, J. Phys. B 21, L31 (1988).

[3] Y. Liang, S. Augst, S. L. Chin, Y. Beaudoin, and M. Chaker, J. Phys. B 27, 5119 (1994).

[4] R. A. Ganeev, Phys. Usp. 52, 55 (2009).

[5] P. B. Corkum, Phys. Rev. Lett. 71, 1994 (1993).

[6] M. Lewenstein, P. Balcou, M. Y. Ivanov, A. L'Huillier, and P. B. Corkum, Phys. Rev. A 49, 2117 (1994).

[7] Y. Mairesse et al. Science 302, 1540 (2003).

[8] R. Velotta, N. Hay, M. B. Mason, M. Castillejo, and J. P. Marangos, Phys. Rev. Lett. 87, 183901 (2001).
[9] M. Lein, N. Hay, R. Velotta, J. P. Marangos, and P. L. Knight, Phys. Rev. Lett. 88, 183903 (2002).

[10] T. Kanai, S. Minemoto, and H. Sakai, Nature (London) 435, 470 (2005).

[11] C. Vozzi et al., Phys. Rev. Lett. 95, 153902 (2005).

[12] J. Itatani, J. Levesque, D. Zeidler, H. Niikura, H. Pépin, J. C. Kieffer, P. B. Corkum, and D. M. Villeneuve, Nature (London) 432, 867 (2004).

[13] S. Haessler et al., Nature Phys. 6, 200 (2010).

[14] B. K. McFarland, J. P. Farrell, P. H. Bucksbaum, and M. Gühr, Science 322, 1232 (2008). 
[15] O. Smirnova, Y. Mairesse, S. Patchkovskii, N. Dudovich, D. Villeneuve, P. Corkum, and M. Y. Ivanov, Nature (London) 460, 972 (2009).

[16] Y. Mairesse et al., Phys. Rev. Lett. 104, 213601 (2010).

[17] A.-T. Le, R. R. Lucchese, S. Tonzani, T. Morishita, and C. D. Lin, Phys. Rev. A 80, 013401 (2009).

[18] C. D. Lin, A.-T. Le, Z. Chen, T. Morishita, and R. Lucchese, J. Phys. B 43, 122001 (2010).

[19] J. W. Cooper, Phys. Rev. 128, 681 (1962).

[20] H. J. Wörner, H. Niikura, J. B. Bertrand, P. B. Corkum, and D. M. Villeneuve, Phys. Rev. Lett. 102, 103901 (2009).

[21] U. Fano, Phys. Rev. 124, 1866 (1961).

[22] G. Raşeev, B. Leyh, and H. Lefebvre-Brion, Z. Phys. D 2, 319 (1986).

[23] F. Keller and H. Lefebvre-Brion, Z. Phys. D 4, 15 (1986).

[24] M. Ya Amusia and J.-P. Connerade, Rep. Prog. Phys. 63, 41 (2000).

[25] M. B. Gaarde and K. J. Schafer, Phys. Rev. A 64, 013820 (2001).

[26] C. Figueira de Morisson Faria, R. Kopold, W. Becker, and J. M. Rost, Phys. Rev. A 65, 023404 (2002).

[27] R. Taïeb, V. Veniard, J. Wassaf, and A. Maquet, Phys. Rev. A 68, 033403 (2003).

[28] H. G. Muller and F. C. Kooiman, Phys. Rev. Lett. 81, 1207 (1998).
[29] D. B. Milošević, J. Phys. B 40, 3367 (2007).

[30] I. A. Ivanov and A. S. Kheifets, Phys. Rev. A 78, 053406 (2008),

[31] D. B. Milošević, Phys. Rev. A 81, 023802 (2010).

[32] I. A. Kulagin and T. Usmanov, Opt. Lett. 34, 2616 (2009).

[33] V. Strelkov, Phys. Rev. Lett. 104, 123901 (2010).

[34] M. B. Gaarde, P. Antoine, A. L'Huillier, K. J. Schafer, and K. C. Kulander, Phys. Rev. A 57, 4553 (1998).

[35] M. D. Feit, J. A. Fleck Jr., and A. Steiger, J. Comput. Phys. 47, 412 (1982).

[36] J. A. Fleck Jr., J. R. Morris, and M. D. Feit, Appl. Phys. 10, 129 (1976).

[37] R. Kosloff and H. Tal-Ezer, Chem. Phys. Lett. 127, 223 (1986).

[38] L. D. Landau and E. M. Lifshitz, Quantum Mechanics: NonRelativistic Theory, 2nd ed. (Pergamon, Oxford, 1965).

[39] D. Gabor, J. Inst. Electr. Eng. 93, 429 (1946).

[40] C. C. Chirilă, I. Dreissigacker, E. V. van der Zwan, and M. Lein, Phys. Rev. A 81, 033412 (2010).

[41] Anh-Thu Le, T. Morishita, and C. D. Lin, Phys. Rev. A 78, 023814 (2008).

[42] C. Jin, A. T. Le, and C. D. Lin, Phys. Rev. A 79, 053413 (2009).

[43] P. Antoine, A. L'Huillier, and M. Lewenstein, Phys. Rev. Lett. 77, 1234 (1996). 